\title{
Ulzeröse Stomatitis und Urethritis mit Augenrötung - Morbus Behçet?
}

\section{Anamnese}

Der 14-jährige Patient erkrankte akut mit Husten und Halsschmerzen, 2 Tage später traten fieberhafte Temperaturen bis $40,6^{\circ} \mathrm{C}$ und Bläschen im Mund auf. Bei Verschlechterung des Allgemeinzustandes und Erbrechen erfolgte am 4. Erkrankungstag die Hospitalisierung des Patienten.

Im externen Krankenhaus zeigten sich eine


eine Konjunktivitis mit Chemosis beidseits ( Abb. 2), Schleimhautulzerationen der Glans penis, Papeln im Bereich des Skrotums ( $\triangleright$ Abb. $\mathbf{3}$ ) sowie einzelne noduläre erythematöse Effloreszenzen an den Extremitäten ( $\triangleright$ Abb. 4). Weiterhin bestand ein produktiver Husten bei jedoch unauffälligem Auskultationsbefund.

\section{Eigenanamnese}

Bereits 2 Jahre (27 Monate) zuvor Hospitalisierung bei ausgeprägter Gingivostomatitis mit Nahrungsverweigerung und Gewichtsabnahme. Die damals durchgeführte Erregerdiagnostik (HSV-, Coxsackie-, ECHOVirus-Serologie) war unauffällig.

Im Kleinkindalter bestand fraglich eine Psoriasis, im Vorschulalter vorübergehende Knie- und Sprunggelenkarthralgien ohne wegweisenden Befund in der kinderrheumatologischen Abklärung sowie Kopfschmerzen. Die sonstige Eigenanamnese ist unauffällig.

\section{Initiale Therapie}

Im externen Krankenhaus erfolgten eine antibiotische Behandlung mit Amoxicillin/Clavulansäure i. v., eine bilanzierte Infusionstherapie, initial die Gabe von Proculin-Augentropfen bzw. im Verlauf Prednisolon-Augentropfen. Zudem erfolgte die orale Gabe von Aciclovir über 5 Tage, später die systemische Gabe von Prednisolon (3 mg/kg KG/Tag i. v.). Unter diesen und zusätzlich supportiven Maßnahmen, u.a. mit diversen Mundspülungen und der lokalen Pflege mit Bepanthen, konnte eine diskrete Besserung des Schleimhautbefundes und des Allgemeinzustands erreicht werden, Entfieberung am 4. Behandlungstag.

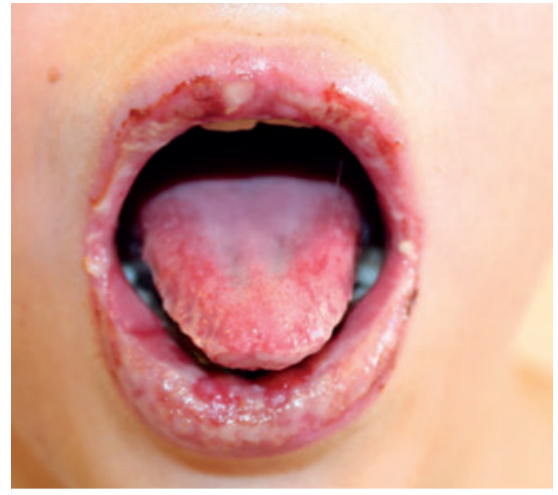

Abb. 1 Ulzeröse Stomatitis mit Beteiligung der Lippen (ca. 14. Erkrankungstag).

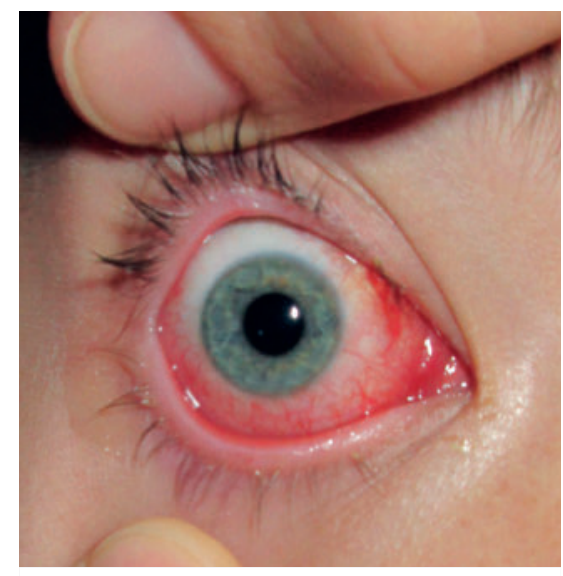

- Abb. 2 Konjunktivitis mit ausgeprägter Chemosis und Hyperämie (ca. 4. Erkrankungstag).

\section{Diagnostik}

\section{Auswärtige Diagnostik}

Die auswärtige Diagnostik ergab folgende Befunde:

- unauffällige kraniale MRT

- Röntgen-Thorax: Infekthili beidseits

- Standortflora im Rachen- und Augenabstrich

- Infektionsserologie:

- HSV-1/2-Ak negativ,

- EBV-Ak negativ,

- ECHO-Virus-Ak negativ,

- Bartonella henselae-Ak negativ,

- Coxsackievirus-Ak: grenzwertig erhöhter IgG-Titer,

- Influenza-B-Ak: durchgemachte Infektion, Influenza-A-Ak: negativ,

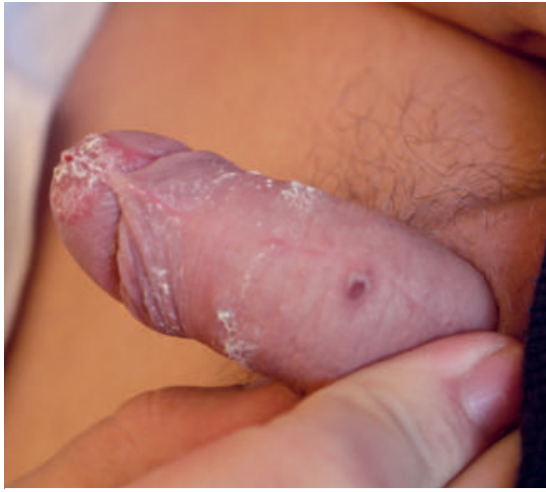

- Abb. 3 Diskrete Schleimhautulzerationen der Glans penis und rötlich-erhabene Papel am Penisschaft (ca. 14. Erkrankungstag).

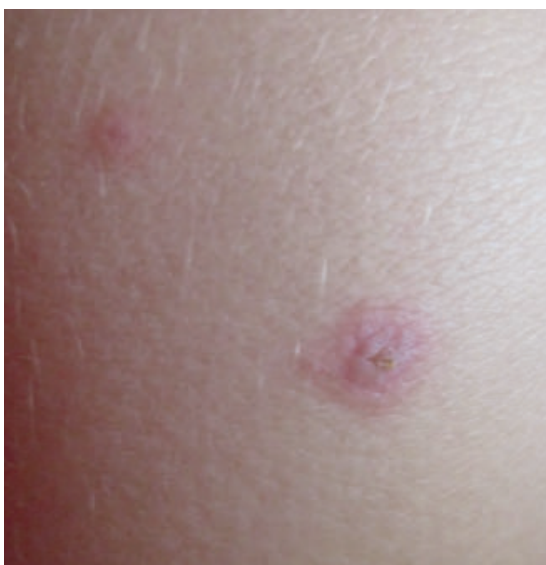

- Abb. 4 Exanthem: papulo-noduläre, erythematöse Effloreszenzen (ca. 4. Erkrankungstag).

- Adenovirus-Ak: durchgemachte Infektion (IgG deutlich erhöht, IgA unauffällig),

- Mykoplasma pneumoniae-Ak: IgG deutlich erhöht, IgA und IgM unauffällig.

- Pathergie-Test negativ,

- Augenarzt: bds. massive konjunktivale und episklerale Injektion, Vorderkammer sauber, Fundus in Miosis regelrecht.

Aufgrund der aktuellen klinischen Befunde und der Vorgeschichte mit ausgeprägter Gingivostomatitis wurde der V.a. einen Morbus Behçet gestellt und es erfolgte die Verlegung in unsere Klinik zur rheumatologischen Diagnostik. 
Bei Aufnahme präsentierte sich der Patient weiterhin mit ausgeprägten aphthösen Schleimhauterosionen v. a. der hochroten Mundschleimhaut, seröser Konjunktivitis, dezenten Ulzera der Penisspitze, periurethraler Rötung mit urethraler Sekretion und extremitätenbetontem Exanthem mit linsengroßen, lividen Papeln. Zudem bestand weiterhin ein produktiver Husten.

\section{Labordiagnostik (SI-Einheiten)}

- CrP 14,4 mg/l, BSG 23/50 mm. Blutbild mit geringer Thrombozytose $\left(425 \times 10^{3} / \mu l\right)$, sonst normwertig. Im Differenzialblutbild Lymphopenie (10\%).

- Transaminasen, Elektrolyte, Nierenwerte, CK und LDH unauffällig. Lipase mit 0,08U/l erniedrigt.

- 24h-Sammelurin auf Protein und Creatinin-Clearance unauffällig.

- Gerinnungsdiagnostik einschließlich v.-Willebrand-Diagnostik unauffällig.

\section{Infektionsdiagnostik}

- Multiplex-PCR-Rachenabstrich: Nachweis von Mykoplasma-pneumoniae-DNA

- Serologie: Mykoplasma-pneumoniae-Ak: IgG, IgA und IgM erhöht $\rightarrow$ Nachweis einer frischen Mykoplasmen-Infektion.

- Leptospiren-Ak negativ. Chlamydia-psittaci- und -trachomatis-Ak negativ. Ureaplasma-urealyticum im Urin negativ. Quantiferon-Test negativ. ASL-Titer: 27 IE/ml (im NB)

- Mikrobiologie: Blutkultur negativ, Abstriche von Mund- und Genitalbereich mit Standortflora.

\section{Immunologische Diagnostik}

- ANA-Titer: 1:320

- ENA-Screening (Jo-1, Scl-70, SSA, SSB, SmD, U1-snRNP): negativ

- Serum-Amyloid A: 234 mg/l (NB<6,4 mg/l), S100 A8/A9 Protein: $17820 \mathrm{ng} / \mathrm{ml}$ (NB:<2940 ng/ $\mathrm{ml}$ ) - > passend zu systemischer Inflammation

- HLA-B51: negativ (Risikomerkmal M. Behçet)

- HLA-B27: negativ

- C3/C4-Komplement: C3 1,92 g/l (NB 0,9-1,8 g/l), C4 im Normbereich

- IgA-Gesamt mit 0,32 erniedrigt, IgM und IgG im Normbereich
- Lymphozyten-Subpopulationen: Absoluter und relativer Anteil der NK-Zellen vermindert: NK-Zellen absolut

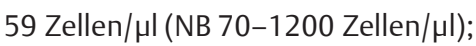
NK-Zellen relativ $3 \%$ (NB 6-27\%); geringe Erhöhung der relativen B-Lymphozytenpopulation: 30\% (NB 8-24\%). T-Lymphozyten mit Subpopulationen unauffällig

- Geringe Monozytose: 13\% (NB 2-11\%). Geringe Thrombozytose $482 \times 10^{3} / \mu \mathrm{l}\left(\mathrm{NB} 175-368 \times 10^{3} / \mu \mathrm{l}\right)$; Beurteilung: Verminderung der NKassoziierten Abwehr

\section{Außerdem:}

- Pathergie-Test erneut negativ

- Hautstanzbiopsie: intrakorneale, granulozytär durchsetzte Serumkruste und minimale oberflächlich-perivasale chronische Entzündung, keine vaskulitischen Veränderungen

- Augenärztliches Konsil: Vorderkammer sauber; Fundus links stärkere Gefäßschlängelung, sonst unauffällig; Diagnose: Verdacht auf rückläufige Episkleritis

\section{Therapie}

Es erfolgte die Schmerzmedikation mit Ibuprofen und Metamizol. Die Gabe von Prednisolon-Augentropfen wurde fortgesetzt. Die genitalen Läsionen besserten sich unter Pflege mit Tannosynth ${ }^{\circledR}$ Lotio. Die Läsionen der Mundschleimhaut zeigten sich zunächst therapieresistent; Kamillan ${ }^{\circledR}$-Mundspülungen, sowie Sucralfat erbrachten keine wesentliche Besserung. Ein Versuch mit einer Prednisolon-haltigen Haftcreme leitete schließlich die Befundbesserung ein.

Die Ernährung erfolgte oral, mit gekühlter, flüssig-breiiger Kost. Die Bronchitis mit produktivem Husten behandelten wir unterstützend mit Kochsalzinhalationen. Nach Nachweis einer akuten Mykoplasmen-Infektion (Serologie und PCR) begannen wir eine orale systemische Therapie mit Clarithromycin.

Letztlich sahen wir insgesamt eine Beschwerdebesserung ab dem 15. Behandlungstag. Wir sahen den Patienten nochmals am 22. Behandlungstag (25. Erkrankungstag) mit nun fast vollständiger Beschwerdefreiheit.

\section{DIAGNOSEKRITERIEN}

M. BEHÇET

Diagnosekriterien $\mathrm{M}$. Behçet nach ICBD von 2010 [8]: $\geq 4$ Punkte notwendig

- Rekurrierende orale Aphtose (innerhalb von 12 Monaten mindestens 3-malig) - 2 Punkte

- Rekurrierende genitale Läsionen - aphtöse Ulzerationen oder Vernarbungen - 2 Punkte

- Okulärer Befall (anteriore oder posteriore Uveitis, retinale Vaskulitis (ophtalmologisch bestätigt) - 2 Punkte

- Hautläsionen (Erythema nodosum, Pseudofollikulitis, papulopustulöse Läsionen oder akneiforme Knötchen bei postpubertären Patienten) - 1 Punkt

- Neurologische Manifestationen - 1 Punkt

- Vaskuläre Manifestationen (Venöse/arterielle Thrombosen, arterielle Aneurysmen) - 1 Punkt

- Positiver Pathergie-Test optional

Pädiatrische Diagnosekriterien M. Behçet (PEDBD 2015) [9]: $\geq 3$ von 6 Kriterien notwendig

- Orale Aphtose ( $\geq 3 /$ Jahr)

- Genitale Läsionen

- Hautmanifestationen

- Neurologische Manifestationen

- Typische Augenbeteiligung (siehe Diagnosekriterien oben nach ICBD)

- Vaskuläre Manifestationen

\section{Bewertung}

1. Die diagnostische Herausforderung im vorliegenden Fall war die bereits zum 2. Mal aufgetretene Symptomatik mit ausgeprägter Gingivostomatitis, aktuell begleitet von genitalen Ulzera und einer Augenbeteiligung. Diese Trias ließ zunächst an die seltene Differenzialdiagnose eines Morbus Behçet denken. Eine Übersicht über mögliche Symptome beim M. Behçet zeigt $\gg$ Tab. 1. Unser Patient erfüllte hiervon das Vorliegen von oralen UIzerationen, genitalen Ulzerationen sowie einer Hautbeteiligung. Schein- 
\Tab.1 Mögliche Symptome bei Morbus Behçet und Mykoplasmen-induzierter Mukositis.

\begin{tabular}{|c|c|c|}
\hline & Morbus Behçet & Mykoplasmen-induzierte Mukositis [6] \\
\hline Augenbeteiligung & $\begin{array}{l}\text { 85-93\% Männer, 67-73\% Frauen; } \\
\text { Panuveitis und/oder retinale Vaskulitis, Hypopyoniritis, } 25 \% \text { Papillitis }\end{array}$ & Ca. $97 \%$ Konjunktivitis \\
\hline Hautbeteiligung & $\begin{array}{l}\text { Insgesamt ca. } 80 \% \text {, v. a. Papulopusteln, auch multiple Erythema nodosa, } \\
\text { selten palpable Purpura, Ulzera }\end{array}$ & Ca. $31 \%$ leichte Hautbeteiligung \\
\hline Orale Aphthen & ca. $98 \%$, rezidivierend (alle 4-12 Wochen) & $\begin{array}{l}\text { Schwere Mundschleimhautläsionen, } \\
\text {-nekrosen (bis 100\%) }\end{array}$ \\
\hline Genitale Aphthen & Ca. $80 \%$, davon $50 \%$ vernarbend & $78 \%$ urogenitale Schleimhautläsionen \\
\hline $\begin{array}{l}\text { Vaskuläre } \\
\text { Manifestationen }\end{array}$ & $\begin{array}{l}15-50 \% \text {, V. a. Thrombophlebitiden, seltener venöse Thrombosen, seltener } \\
\text { arterielle Aneurysmen oder sehr selten arterielle Gefäßverschlüsse }\end{array}$ & Keine \\
\hline Gelenkbeteiligung & Ca. $50 \%$, v. a. periphere Oligoarthritis der unteren Extremität & $\begin{array}{l}\text { Eher nein bei MPAM, aber generell bei Mykoplasmen- } \\
\text { infektion Arthralgien, (Poly)arthritis möglich }\end{array}$ \\
\hline Genetische Marker & $\begin{array}{l}\text { HLA-B51 (50-80\%, OR 5-7) } \\
\text { (HLA-A26; OR 2) } \\
\text { (IL-10 und IL-23R-IL-12RB2, OR 1,45) }\end{array}$ & Keine bekannt \\
\hline Sonstige Marker & Pathergiephänomen: in 15-60\% & Serologie, PCR; oft hohe BSG \\
\hline Histologie & Leukozytoklastische Vaskulitis & \\
\hline Sonstiges & $\begin{array}{l}\text { Selten: Epididymitiden, gastrointestinale Ulzera, ZNS-Beteiligung } 10 \% \\
\text { (Sinusvenenthrombose, Meningoenzephalitis, parenchymatöse Vaskulitis } \\
\text { mit Apoplex) }\end{array}$ & $\begin{array}{l}\text { Bis } 100 \% \text { : Atemwegsinfektion und Fieber vor (!) Auf- } \\
\text { treten von Mundschleimhaut- und Augenbeteiligung; } \\
\text { radiologisch Zeichen einer Pneumonie }\end{array}$ \\
\hline
\end{tabular}

bar lag auch eine zum M. Behçet passende Augenbeteiligung vor; ein wichtiger Unterschied hier ist jedoch, dass beim M. Behçet in der Regel eine retinale Vaskulitis oder (Pan-)Uveitis vorliegt, während unser Patient primär eine Konjunktivitis aufwies.

Dennoch war auch für uns ein M. Behçet zunächst eine differenzialdiagnostische Überlegung, sodass die oben aufgeführte Immundiagnostik sowie ergänzend die Biopsie einer Hautläsion erfolgte. Hierbei ergab sich primär der Nachweis einer schweren Entzündungsreaktion ohne Hinweis auf eine Vaskulitis. Im Augenärztlichen Konsil konnten eine Uveitis oder Vaskulitis ausgeschlossen werden.

Retrospektiv waren die diagnostischen Kriterien für einen $\mathrm{M}$. Behçet, wie nach den pädiatrischen Diagnosekriterien von 2015 (PEDBD 2015) gefordert, nicht erfüllt (siehe Kasten).

Gefordert wird hier im Rahmen der Kriterien, dass die oralen Aphthen mindestens dreimalig innerhalb eines Zeitraumes von 12 Monaten rezidivieren und dass die Augenbeteiligung in Form einer Uveitis oder retinalen Vaskulitis vorliegt. Da bei unserem Patienten weder eine neurologische Beteiligung noch eine vaskuläre Beteiligung vorla- gen, waren als geforderte Diagnosekriterien lediglich die genitalen Ulzerationen sowie eine Hautbeteiligung vorliegend und damit nur 2 der mindestens geforderten 3 Kriterien erfüllt. Das gemeinsame Auftreten von Konjunktivitis, Ulzerationen im Bereich der Glans penis und im Mundbereich sowie einer Hautbeteiligung kann auch an extraartikuläre Manifestationen im Rahmen einer reaktiven Arthritis denken lassen [10]. Selten kann diese - wie bei unserem Patienten vorliegend - durch Atemwegsinfekte ausgelöst werden. Jedoch zeigte unser Patient zu keinem Zeitpunkt Gelenkschmerzen oder weitere Zeichen einer Gelenkentzündung.

2. Infektionen mit Mykoplasma pneumoniae können häufig mit einem Exanthem, seltener auch mit Enanthem einhergehen. Teilweise wurden diese in der Vergangenheit als Stevens-Johnson-Syndrom, inkomplettes Stevens-Johnson-Syndrom, Fuchs-Syndrom oder Erythema multiforme klassifiziert, inzwischen immer mehr als eigene, wenngleich pathogenetisch möglicherweise verwandte, Entität („Mycoplasma pneumoniae associated mucositis“, „MPAM“) [1, 5, 6]. Es erkranken hier v. a. Schulkinder (Me- dian 13,5 Jahre), mit einer hohen Jungenwendigkeit (26/6) [6]. Die genaue Pathogenese der Erkrankung ist unklar, eine genetische Prädisposition wird vermutet.

Irreführend in unserem Fall war die bereits vorliegende Mykoplasmen-Serologie mit erhöhtem IgG-Titer, aber unauffälligem IgA- und IgM-Titer, welche eine abgelaufene Mykoplasmen-Infektion vermuten ließ. Mykoplasmen-IgM-Ak treten ca. 7-10 Tage nach Beginn einer frischen Mykoplasmen-Infektion auf [2]. Da der Patient am 4. Tag der Erkrankung hospitalisiert wurde, wurden im externen Krankenhaus noch keine IgM-Ak nachgewiesen. Mykoplasmen-lgG-Ak persistieren für 2-9 Jahre, fallen jedoch bei lediglich stattgehabtem Infekt der oberen Atemwege (keine Pneumonie) nach 2 Jahren wieder ab, sodass eine Re-Infektion möglich wird. Im Falle einer Re-Infektion ist eine direkte IgG-Antwort, vor Auftreten von IgM-Ak, möglich [2]. Im vorliegenden Fall gehen wir anhand der zeitversetzt abgenommenen serologischen Diagnostik daher von einer Zweitinfektion mit Mykoplasmen aus, welche die aktuelle Symptomatik auslöste.

Es kann nur spekuliert werden, ob bereits die erste beschriebene Gingivostomatitis 
Folge einer Infektion mit Mykoplasmen war, oder ob die Erstinfektion zu einem anderen Zeitpunkt auftrat.

Prinzipiell ist in der Literatur ein Wiederauftreten von Haut- und Schleimhautläsionen bei Mykoplasmen-Re-Infektionen beschrieben [7]; eine individuelle Prädisposition ist zu vermuten. Bei unserem Patienten konnten wir als einzige Auffälligkeiten in der Immundiagnostik einen IgA-Mangel sowie eine verminderte NK-assoziierte Abwehr feststellen. Die häufigste Ursache einer transienten Verminderung der NK-Zellen ist neben akutem Stress bzw. Krankheit eine systemische Glukokortikoidbehandlung, sodass auch in unserem Fall nicht von einem Immundefekt ausgegangen werden muss. Zu den bisher beschriebenen Fällen mit MPAM sind keine weiteren immunologischen Parameter bekannt. Die Pathogenese von MPAM bedarf weiterer Forschung.

Aufgrund weniger vorliegender Fälle gibt es keine einheitliche Therapieempfehlung für MPAM. Therapeutisch scheint die systemische Gabe von Kortikosteroiden einen positiven Effekt zu haben - auch unser Patient berichtete von einer Besserung nach systemischer Prednisolon-Gabe. Ebenso wird die Gabe von Immunglobulinen erwähnt, wobei hier weniger Einzelfälle beschrieben sind.

Bei gesicherter Infektion werden zudem meist Makrolide gegeben; unklar ist hierbei, ob diese noch einen Einfluss auf den Verlauf der Schleimhaut- und Hautbeteiligung nehmen. Nach Diagnosestellung erhielt auch unser Patient Clarithromycin; ob dies oder die supportiven Maßnahmen die Besserung einleiteten oder sich hier letztlich der natürliche Verlauf der Erkrankung zeigte, kann nicht sicher abgegrenzt werden.

\section{FAZIT}

1. Die Mykoplasma-pneumonia-assoziierte Mukositis mit/ohne Hautbeteiligung ist eine seltene, aber schwer beeinträchtigende Komplikation einer akuten Mykoplasmen-Infektion. Bei schwerer Mukositis mit vorangegangenen Prodromi/Begleiterscheinungen im Sinne von Fieber und Symptomen eines Atemwegsinfektes sollte differenzialdiagnostisch daran gedacht werden.

2. Die alleinige Serologie ist für die Diagnose einer akuten Mykoplasmen-Infektion unzureichend [2]. IgM-Ak steigen oft erst nach 7 Tagen an, eine Re-Infektion ist in frühen Stadien serologisch kaum von einer abgelaufenen Infektion zu unterscheiden. Gleichzeitig hat die zweite etablierte diagnostische Methode - die Mykoplasmen-PCR aus dem Rachenabstrich - geringere Sensitivitätsraten von lediglich 78-92\% und kann zudem kaum zwischen Besiedelung und akuter Infektion unterscheiden [2]. Empfehlenswert zur Diagnostik be V.a. akute Mykoplasmen-Infektion ist daher die Kombination aus Serologie mit lgM-Ak und Mykoplasmen-PCR im Rachenabstrich. Bei negativer initialer Diagnostik und fortbestehendem Verdacht sollte eine serologische Kontrolle im zeitlichen Verlauf erfolgen.

3. Einheitliche Therapierichtlinien für die Mykoplamen-assoziierte Mukositis fehlen bislang. Hier bedarf es weiterer Studien.

Interessenkonflikt

Die Autoren geben an, dass kein Interessenkonflikt besteht.

\section{Einhaltung ethischer Richtlinien}

Eine schriftliche Einwilligung des Patienten/ der Eltern des Patienten zur Veröffentlichung der Kasuistik liegt vor.

\section{Autoren}
A. Markowsky'1, M. Pommerenke², D. Gutmann ${ }^{3}$, D. Windschall ${ }^{2}$
${ }^{1}$ Klinik für Kinder- und Jugendmedizin, Asklepios Klinik Weißenfels
${ }^{2}$ Klinik für Kinder- und Jugendmedizin, St. Georg Klinikum Leipzig
${ }^{3}$ Abteilung für Kinder- und Jugendmedizin, Klinikum Burgenlandkreis gGmbH, Georgius-Agricola-Klinikum Zeitz

Korrespondenzadresse

Priv.-Doz. Dr. Daniel Windschall

St. Georg Klinikum Leipzig

Delitzscher Straße 141, 04129 Leipzig

danwindschall@web.de

Literatur

[1] Canavan TN, Mathes EF, Frieden I, Shinkai $\mathrm{K}$. Mycoplasma pneumoniae induced rash and mucositis as a syndrome distinct from Stevens-Johnson syndrome and erythema multiforme: A systematic review. J Am Acad Dermatol 2015; 72(2): 239-245

[2] Ferwerda A, Moll H. de Groot R. Respiratory tract infections by Mycoplasma pneumoniae in children: a review of diagnostic and therapeutic measures. Eur ] Pediatr 2001; 160: 483-491

[3] Kötter I, Xenitidis T, Fierlbeck $G$ et al. Morbus Behçet. Z Rheumatol 2012; 71: 685-697

[4] Altenburg A, Mahr A, Maldini C et al. Epidemiologie und Klinik des Morbus Adamantiades-Behçet in Deutschland. Der Ophthalmologe 2012; 109: 531-541

[5] Vujic I, Shroff A, Grzelka M et al. Mycoplasma pneumoniae-associated mucositis - case report and systematic review of literature. JEADV 2015; 29: 595-598

[6] Meyer Sauteur PM, Goetschel P, Lautenschlager S. Mycoplasma pneumoniae and mucositis - part of the Stevens-Johnson syndrome spectrum. J Deutsch Dermatol Ges 2012; 10: 740-745

[7] Finkelstein Y, Soon GS, Acuna P et al. Recurrence and Outcomes of Stevens-Johnson Syndrome and Toxic Epidermal Necrolysis in Chilrden. Pediatrics 2011; 128: 723-728

[8] Batu ED. Diagnostic/classification criteria in pediatric Behçet’s Disease. Rheumatology International 2018; 39: 37-46

[9] Koné-Paut I. Behçet's Disease in children, an overview. Pediatric Rheumatology 2016; 14: 10

[10] Rihl M. Update zur reaktiven Arthritis. Z Rheumatol 2016; 75: 869-877

\section{Bibliografie}

DOI https://doi.org/10.1055/a-0886-5997 arthritis + rheuma 2019; 39: 261-265 (c) Georg Thieme Verlag KG Stuttgart - New York ISSN 0176-5167 